\title{
A Case of Monotone Ratio Growth for Quadratic-Like Mappings
}

by

\author{
Waldemar PAŁUBA \\ Presented by Andrzej LASOTA
}

\begin{abstract}
Summary. This is a study of the monotone (in parameter) behavior of the ratios of the consecutive intervals in the nested family of intervals delimited by the itinerary of a critical point. We consider a one-parameter power-law family of mappings of the form $f_{a}=-|x|^{\alpha}+a$. Here we treat the dynamically simplest situation, before the critical point itself becomes strongly attracting; this corresponds to the kneading sequence $R R R .$. , or - in the quadratic family - to the parameters $c \in[-1,0]$ in the Mandelbrot set. We allow the exponent $\alpha$ to be an arbitrary real number greater than 1 .
\end{abstract}

1. Introduction. The question of monotonicity of the dynamics in a one-parameter family of maps of an interval has long been one of the crucial problems in the field. A remarkable progress in this research has been achieved in recent years (see the reference list). However, usually the quasiconformal technics are involved in those proofs, requiring the maps in question to admit complex analytic extensions. On the other hand, numerical evidence does not suggest any dramatic breakdown if we consider a noninteger exponent, say 1,99, instead. In this work we present an argument that solves the simplest monotonicity problem for an arbitrary real power-law family.

Consider a one-parameter family of mappings of the form $f_{a}=-|x|^{\alpha}+a$, with $\alpha>1$ and a real positive parameter $a$. Let $x_{a}$ be the positive fixed point of $f_{a}$. It is clear that when we increase the parameter $a$ the absolute value of the eigenvalue at the fixed point $x_{a}$ grows, because $|x|^{\alpha}$ is a convex map.

2000 Mathematics Subject Classification: Primary 37D05.

Key words and phrases: quadratic-like mapping of an interval, post-critical orbit, Poincaré length, nonlinearity, Poincaré push.

Supported by the KBN grant 2 P03A 02208. 
In this note our approach is to look at the behavior of the whole postcritical orbit rather than at the eigenvalue directly.

Suppose we are given a map $f_{a}$ with kneading sequence $R R R \ldots$, so that the orbit of the critical point tends to a fixed point or to a period 2 attractor. For the quadratic family this means the Mandelbrot set address $c$ is in the interval $[-1,0]$. This post-critical orbit delimits a sequence of nested intervals where any two consecutive intervals share one endpoint.

Our objective is to establish that for any two such successive intervals, the proportion of their lengths is an increasing function of the parameter $a$.

Notice that unlike the eigenvalue statement, this is not a consequence of mere convexity, not even in the simplest case of an attracting fixed point. Actually, the growth of the absolute value of the multiplier corresponds to the increase of the ratio of very short intervals around the fixed point, where the map acts almost linearly. What we claim here is that not only does the "ultimate" ratio grow but also all the length ratios along the itinerary of the critical point do so simultaneously.

To prove our claim we shall begin with an overview of some properties of the mapping $|x|^{\alpha}$ viewed in the Poincaré metric. In the proofs below we essentially use the fact that the maps we deal with are not just negative Schwarzian maps, but specific homogeneous maps. This will provide more subtle tools than the distortion estimates alone.

Acknowledgements. The author would like to thank the referee for his thorough and detailed report that led to the improvement of the exposition.

2. The Poincaré geometry of an interval. We begin with some notation. The Poincaré coordinate of a point $x$ on the positive half-axis $(0, \infty)$ is defined by

$$
p(x)=\ln x
$$

The Poincaré coordinate of a point $x$ in an open, oriented interval $(a, b)$ is defined by

$$
p(x)=\ln \frac{x-a}{b-x} .
$$

The Poincaré length of an interval $(x, y)$ contained in $(a, b)$ is by definition $|p(x)-p(y)|$.

We shall write $p_{a b}(x)$ instead of $p(x)$ whenever any confusion as to the domain interval concerned might arise.

From now on we fix a real number $\alpha>1$ and the symbol $h$ will stand for the power-law mapping of the positive half-axis $\mathbb{R}_{+}$onto itself,

$$
h(x)=x^{\alpha} \quad \text { with } x \in(0, \infty), \alpha \in(1, \infty) .
$$

For a given map $h: \mathbb{R}_{+} \rightarrow \mathbb{R}_{+}$, or its restriction to an interval $(a, b) \subset$ $(0, \infty)$, i.e. $\left.h\right|_{(a, b)}:(a, b) \mapsto\left(a^{\alpha}, b^{\alpha}\right)$, we shall be considering its Poincaré 
counterpart $\bar{h}: \mathbb{R} \rightarrow \mathbb{R}$, i.e. the mapping defined by the formula

$$
\bar{h}_{a b}(t)=p_{h(a) h(b)}\left(h\left(p_{a b}^{-1}(t)\right) .\right.
$$

This "bar" notation for the Poincaré counterpart of a homeomorphism of an interval will also be kept for maps other than a restriction of $h$.

We start with the following immediate observation.

Proposition 2.1. The mapping $\bar{h}_{0 \infty}$ is a linear map satisfying

$$
\bar{h}(t)=\alpha t
$$

Proof. By the very definition of $\bar{h}$ and of the Poincaré coordinate on the half-axis we have $\bar{h}_{0 \infty}(t)=p_{0 \infty}\left(h\left(p_{0 \infty}^{-1}(t)\right)\right)=\ln \left(e^{t}\right)^{\alpha}=\alpha t$.

In other words:

For any $a, b, c \in \mathbb{R}_{+}$the quantity $(\ln c-\ln b) /(\ln b-\ln a)$ is preserved under the mapping $x \mapsto x^{\alpha}$.

Now we consider an orientation preserving diffeomorphism $\varphi:(a, b) \rightarrow$ $(c, d)$, and assume that there exist finite or infinite limits $\varphi^{\prime}(a)=\lim _{x \rightarrow a} \varphi^{\prime}(x)$ as well as $\varphi^{\prime}(b)=\lim _{x \rightarrow b} \varphi^{\prime}(x)$. We define

$$
\begin{aligned}
& \bar{\varphi}^{+}=\ln \frac{d-c}{(b-a) \varphi^{\prime}(b)}=\lim _{t \rightarrow+\infty}(\bar{\varphi}(t)-t), \\
& \bar{\varphi}^{-}=\ln \frac{\varphi^{\prime}(a)(b-a)}{d-c}=\lim _{t \rightarrow-\infty}(\bar{\varphi}(t)-t) .
\end{aligned}
$$

As usual, $\bar{\varphi}$ stands here for the Poincaré counterpart of $\varphi$. The numbers $\bar{\varphi}^{+}, \bar{\varphi}^{-}$can be thought of as the limit values of the "push" (in the Poincaré coordinates, of course) towards the endpoints, generated by $\varphi$.

In what follows, by the Poincaré push of a diffeomorphism $\varphi:(a, b) \rightarrow$ $(c, d)$ at a point $x \in(a, b)$ we shall mean the quantity

$$
p_{c d}(\varphi(x))-p_{a b}(x) \text {. }
$$

Its absolute value will be referred to as the strength of the Poincaré push. The limit values at the endpoints, whenever they exist, are also called the Poincaré push at $x=a$ or $x=b$.

In case of the power-law map, due to its homogeneity, we have the following immediate remark.

REMARK 2.1. For a restriction of the power-law map $\varphi=\left.h\right|_{(a, b)}$, the quantities $\bar{\varphi}^{+}, \bar{\varphi}^{-}$depend solely on the Poincaré length of the interval $(a, b)$ on the positive half-axis, i.e. they are determined by the number $p_{0 \infty}(b)-$ $p_{0 \infty}(a)$.

Thus we shall often fix the right endpoints $b=d=1$ and choose the left endpoint $a$ of the domain interval within $(0,1)$. 
In the following, the Poincaré length of an interval $(a, b)$ of the positive half-axis will be referred to as its nonlinearity. This name is justified by the fact that the nonlinearity of the mapping $h$ is the derivative of $\ln h^{\prime}$, so this Poincaré length actually is, up to the multiplicative constant $\alpha$, the integral of the nonlinearity of $h$ over $(a, b)$.

Now we are in a position to state our main lemma.

LEMma 2.1. For any $x, y \in(0,1)$ the following equality holds:

$$
\bar{h}_{x 1}^{-}-\bar{h}_{y 1}^{-}=\left(p_{01}(h(x))-p_{01}(h(y))\right)-\left(p_{01}(x)-p_{01}(y)\right) .
$$

Proof. This can be viewed as a consequence of the linearity of $\bar{h}_{0 \infty}$ (cf. Proposition 2.1), and can be derived from the examination of the change of the infinitesimal element of the Poincaré metric. However, a straightforward calculation yields a much shorter proof:

$$
\begin{aligned}
\bar{h}_{x 1}^{-}-\bar{h}_{y 1}^{-} & =\ln \frac{\alpha x^{\alpha-1}(1-x)\left(1-y^{\alpha}\right)}{\left(1-x^{\alpha}\right) \alpha y^{\alpha-1}(1-y)}=\ln \frac{x^{\alpha}\left(1-y^{\alpha}\right) y(1-x)}{y^{\alpha}\left(1-x^{\alpha}\right) x(1-y)} \\
& =\left(p_{01}(h(x))-p_{01}(h(y))\right)-\left(p_{01}(x)-p_{01}(y)\right) .
\end{aligned}
$$

Roughly speaking, the lemma tells us that while we move from $x$ to $y$ within the interval $(0,1)$, the increase of the strength of the limit Poincaré "push" equals the surplus in the Poincaré coordinate change. Let us remark here that it is vital for Lemma 2.1 to hold that it is the critical point of $h$ (i.e. the point 0 ) which is chosen as one of the endpoints. When this is not the case we need the following generalization:

Proposition 2.2. For any diffeomorphism $\varphi:(a, b) \rightarrow(c, d)$ and any $x \in(a, b)$ we have

$$
p_{c d}(\varphi(x))-p_{a b}(x)=\bar{\varphi}_{x b}^{-}+\bar{\varphi}_{a x}^{+} .
$$

Proof. Again, this is an immediate calculation:

$$
\begin{aligned}
p_{c d}(\varphi(x))-p_{a b}(x) & =\ln \left(\frac{\varphi(x)-c}{d-\varphi(x)} \cdot \frac{b-x}{x-a}\right) \\
& =\ln \left(\varphi^{\prime}(x) \cdot \frac{b-x}{d-\varphi(x)}\right)+\ln \frac{\varphi(x)-c}{(x-a) \varphi^{\prime}(x)} \\
& =\lim _{t \rightarrow-\infty}\left(\bar{\varphi}_{x b}(t)-t\right)+\lim _{t \rightarrow+\infty}\left(\bar{\varphi}_{a x}(t)-t\right) .
\end{aligned}
$$

Notice that for $\varphi=\left.h\right|_{(0,1)}$, because of the homogeneity of $h$, the quantity $\bar{\varphi}_{0 x}^{+}$does not depend on $x$ and is equal to $-\ln \alpha$, so we can also derive Lemma 2.1 as a special case of Proposition 2.2.

Returning to homogeneous maps let us record three more properties.

Proposition 2.3. For any triple of points $0<a<b<c$ we have

$$
\left|\bar{h}_{a c}^{-}-\bar{h}_{b c}^{-}\right|>\left|\bar{h}_{a b}^{-}\right|, \quad\left|\bar{h}_{a c}^{+}-\bar{h}_{a b}^{+}\right|<\left|\bar{h}_{b c}^{+}\right| \text {. }
$$


Proof. Set $a=b \theta$, where $0<\theta<1$. By homogeneity we may set $c=1$. Then

$$
\begin{aligned}
\bar{h}_{a c}^{-}-\bar{h}_{b c}^{-} & =\ln \left(\frac{1-a}{1-a^{\alpha}} \cdot h^{\prime}(a)\right)-\ln \left(\frac{1-b}{1-b^{\alpha}} \cdot h^{\prime}(b)\right) \\
& =\ln \left(\frac{h^{\prime}(a)}{h^{\prime}(b)} \cdot \frac{1-\theta b}{1-b} \cdot \frac{1-b^{\alpha}}{1-(\theta b)^{\alpha}}\right) .
\end{aligned}
$$

For the intervals $(a, c)$ and $(b, c)$ endowed with positive orientation, the quantities $\bar{h}_{a c}^{-}$and $\bar{h}_{b c}^{-}$are both negative, by (2.3). Having set $c=1$, we return to the right hand side of the formula (2.4) of Lemma 2.1 to see that for $a=x, b=y$ we subtract there the Poincaré length of $(h(a), h(b))$ from that of $(a, b)$ (both measured within $(0,1))$, so this difference is negative, because for $\alpha>1$ the mapping $h$ expands the Poincaré metric on $(0,1)$. Thus $\bar{h}_{a c}^{-}-\bar{h}_{b c}^{-}$is negative, which can also be verified by a direct calculation. Therefore to prove that this quantity is smaller than

$$
\bar{h}_{a b}^{-}=\ln \left(h^{\prime}(a) \frac{b-a}{b^{\alpha}-a^{\alpha}}\right)=\ln \left(\frac{h^{\prime}(a)}{b^{\alpha-1}} \cdot \frac{1-\theta}{1-\theta^{\alpha}}\right)
$$

we only need to check that, given $b, \theta \in(0,1)$ and $\alpha>1$, the inequality

$$
\frac{1}{\alpha}\left(\frac{1-\theta b}{1-b}\right) \cdot\left(\frac{1-b^{\alpha}}{1-(\theta b)^{\alpha}}\right)<\frac{1-\theta}{1-\theta^{\alpha}}
$$

holds true. This follows from the homogeneity of $x \mapsto|x|^{\alpha}$. See Lemma 2.2 below.

The other inequality follows in a similar way.

Lemma 2.2. Suppose $x, y \in(0,1)$ and $\alpha>1$. Then

$$
\frac{1}{\alpha} \cdot \frac{\left(1-x^{\alpha}\right)\left(1-y^{\alpha}\right)}{1-(x y)^{\alpha}}<\frac{(1-x)(1-y)}{1-x y} .
$$

Proof. Given $x, y \in(0,1)$, the inequality (2.5) is clearly satisfied when $\alpha$ is large enough, and turns into an equality when $\alpha \rightarrow 1$. Thus it will be enough to show that the left hand side of (2.5) is decreasing in $\alpha$. Indeed, the denominator of its $\alpha$-derivative is always positive, while the numerator is equal to

$$
-(1-v)^{2} u \ln u-(1-u)^{2} v \ln v-(1-u)(1-v)(1-u v),
$$

where we have set $u=x^{\alpha} \in(0,1), v=y^{\alpha} \in(0,1)$, and so we will be done once we show

$$
-(1-v)^{2} u \ln u-(1-u)^{2} v \ln v<(1-u)(1-v)(1-u v) .
$$

Expanding $-\ln u$ and $-\ln v$ into power series, and cancelling out the term 
$(1-u)(1-v)$, we see that $(2.6)$ turns into

$$
\begin{aligned}
u(1-v) & +\frac{1}{2} u(1-u)(1-v)+\frac{1}{3} u(1-u)^{2}(1-v)+\ldots \\
& +v(1-u)+\frac{1}{2} v(1-v)(1-u)+\frac{1}{3} v(1-v)^{2}(1-u)+\ldots<1-u v,
\end{aligned}
$$

which further amounts to

$$
\begin{aligned}
& \frac{1}{2} u(1-u)(1-v)+\frac{1}{3} u(1-u)^{2}(1-v)+\ldots \\
& \quad+\frac{1}{2} v(1-v)(1-u)+\frac{1}{3} v(1-v)^{2}(1-u)+\ldots<(1-u)(1-v) .
\end{aligned}
$$

This can again be divided out by the ubiquitous term $(1-u)(1-v)$, and returning to the closed form for the logarithmic functions on the left hand side we get yet another equivalent form of (2.6):

$$
\frac{u}{(1-u)^{2}}(-\ln u-(1-u))+\frac{v}{(1-v)^{2}}(-\ln v-(1-v))<1 .
$$

Finally, we arrive at an inequality in one variable only:

$$
\frac{u}{(1-u)^{2}}(-\ln u-(1-u))<\frac{1}{2}
$$

or

$$
-u \ln u<\frac{1}{2}\left(1-u^{2}\right),
$$

which is elementarily true for $u \in(0,1)$.

Another observation about $\bar{h}_{a b}$ is that the strength of the Poincaré push is a monotone function and so it is largest at the left endpoint (i.e. the one closer to the critical point). Similarly to the proof of Proposition 2.3 we can establish that the quantities compared in Proposition 2.4 below are all negative. Thus for their absolute values (which we have in mind when talking about the "strength of the push") the opposite inequalities hold. Here is a precise statement.

Proposition 2.4. For any $0<a<x_{2}<x_{1}<b$ we have

$$
\bar{h}_{a b}^{+}>p_{a b}\left(h\left(x_{1}\right)\right)-p_{a b}\left(x_{1}\right)>p_{a b}\left(h\left(x_{2}\right)\right)-p_{a b}\left(x_{2}\right)>\bar{h}_{a b}^{-} .
$$

Proof. Recall that by Proposition 2.2 the Poincaré push equals

$$
\bar{h}_{a b}\left(p_{a b}(x)\right)-p_{a b}(x)=\bar{h}_{x b}^{-}+\bar{h}_{a x}^{+} .
$$

When we move the point $x$ towards the endpoint $a$ we add to the nonlinearity of one interval (i.e. the right hand one) and subtract the same amount from the nonlinearity of the other. By Remark 2.1 and a direct computation we see that for any interval $(r, s)$ of nonzero nonlinearity we have

$$
\left|\bar{h}_{r s}^{-}\right|>\left|\bar{h}_{r s}^{+}\right|
$$


because, again, fixing $u \in(0,1)$ and differentiating in $\alpha$ we can verify that for all $u \in(0,1)$ the following inequality holds:

$$
\frac{h^{\prime}(1)}{\left(\frac{1-h(u)}{1-u}\right)}<\frac{\left(\frac{1-h(u)}{1-u}\right)}{h^{\prime}(u)} .
$$

Moving $x$ from the position $x_{1}$ to $x_{2}$ we juxtapose the intervals $\left(x_{2}, x_{1}\right)$ and $\left(x_{1}, b\right)$. By Proposition 2.3 above we see that the change of the value of $\bar{h}_{x b}^{-}$, i.e. the quantity $\left|\bar{h}_{x_{2} b}^{-}-\bar{h}_{x_{1} b}^{-}\right|$, is greater than the number $\left|\bar{h}_{x_{1} x_{2}}^{-}\right|$ corresponding to the amount of nonlinearity added by moving $x$ toward the critical point. Simultaneously $\bar{h}_{a x}^{+}$changes by at most the quantity $\left|\bar{h}_{x_{1} x_{2}}^{+}\right|$ corresponding to this same nonlinearity (recall that by Remark $2.1, \bar{h}^{+}$and $\bar{h}^{-}$above are well defined). This shows that the Poincaré push is monotone in $p(x)$. The inequalities with the limit values come from letting $x$ tend to either endpoint.

When we increase the nonlinearity of an interval and keep the Poincaré coordinate of a point fixed, we have a similar property:

Proposition 2.5. Suppose that the intervals $(a, b),(c, d) \subset \mathbb{R}_{+}$are so that

$$
p_{0 \infty}(b)-p_{0 \infty}(a)<p_{0 \infty}(d)-p_{0 \infty}(c) .
$$

Then the function $f: \mathbb{R} \rightarrow \mathbb{R}$ given by the formula

$$
f(t)=\left(\bar{h}_{\phi d}^{-}+\bar{h}_{c \phi}^{+}\right)-\left(\bar{h}_{\psi b}^{-}+\bar{h}_{a \psi}^{+}\right),
$$

where $\phi=\phi(t)=p_{c d}^{-1}(t)$ and $\psi=\psi(t)=p_{a b}^{-1}(t)$, is increasing.

Proof. We shall proceed much as in the proofs of Proposition 2.3 and Lemma 2.2 above. Firstly, we examine the following simpler situation. We fix an interval of a given nonlinearity and, by homogeneity, we normalize it to be the interval $(\beta, 1)$. Then we juxtapose $(\beta, 1)$ and an interval $(1, t)$ and let the endpoint $t$ vary. When $t$ increases, then the strength of the Poincaré push measured at $\beta$, i.e. the quantity $\left|\bar{h}_{\beta t}^{-}\right|$, increases. So does the strength of the Poincaré push at 1 , i.e. $\left|\bar{h}_{\beta 1}^{+}+\bar{h}_{1 t}^{-}\right|$. The essential point is that the latter increase is smaller than the former. This is again a consequence of the homogeneity of $h: x \mapsto|x|^{\alpha}$ and is shown separately in Lemma 2.3 below. Similarly, when we keep $t>1$ fixed and let the other endpoint vary, an analogous but opposite inequality between the rates of increase of the strength of Poincaré pushes holds for $\left|\bar{h}_{\beta 1}^{+}+\bar{h}_{1 t}^{-}\right|$and $\left|\bar{h}_{\beta t}^{+}\right|$; this is the other part of the statement of Lemma 2.3.

The statement of Proposition 2.5 easily follows from the observations above. Let us pick $t_{1}<t_{2}$. There exists a unique point $t_{3}$ in $\left(t_{1}, t_{2}\right)$ such that the nonlinearity of the interval $\left(p_{c d}^{-1}\left(t_{1}\right), p_{c d}^{-1}\left(t_{3}\right)\right)$, i.e. the number 
$p_{0 \infty}\left(p_{c d}^{-1}\left(t_{3}\right)\right)-p_{0 \infty}\left(p_{c d}^{-1}\left(t_{1}\right)\right)$, is equal to the nonlinearity of $\left(p_{a b}^{-1}\left(t_{1}\right), p_{a b}^{-1}\left(t_{2}\right)\right)$. The intervals $\left(c, p_{c d}^{-1}\left(t_{1}\right)\right)$ and $\left(p_{c d}^{-1}\left(t_{3}\right), d\right)$ have larger nonlinearities than $\left(a, p_{a b}^{-1}\left(t_{1}\right)\right)$ and $\left(p_{a b}^{-1}\left(t_{2}\right), b\right)$ respectively. This is so because $\left(c, p_{c d}^{-1}\left(t_{1}\right)\right)$ constitutes the same fraction of $(c, d)$ as does $\left(a, p_{a b}^{-1}\left(t_{1}\right)\right)$ within $(a, b)$, and the nonlinearity of $(c, d)$ is larger than that of $(a, b)$; also the fraction constituted by $\left(p_{c d}^{-1}\left(t_{3}\right), d\right)$ within $(c, d)$ is even larger than that of $\left(p_{a b}^{-1}\left(t_{2}\right), b\right)$ within $(a, b)$, because $t_{3}<t_{2}$. It is evident that the same (or even larger) fraction of an interval with greater nonlinearity has itself greater nonlinearity compared to the respective fraction of an interval with smaller nonlinearity.

Applying the first observation derived previously from Lemma 2.3 to the interval $\left(p_{a b}^{-1}\left(t_{1}\right), p_{a b}^{-1}\left(t_{2}\right)\right)$ juxtaposed with $\left(p_{a b}^{-1}\left(t_{2}\right), b\right)$ and the interval $\left(p_{c d}^{-1}\left(t_{1}\right), p_{c d}^{-1}\left(t_{3}\right)\right)$ juxtaposed with $\left(p_{c d}^{-1}\left(t_{3}\right), d\right)$ we see that

$$
\left|\bar{h}_{p_{c d}^{-1}\left(t_{1}\right) d}^{-}-\bar{h}_{p_{a b}^{-1}\left(t_{1}\right) b}^{-}\right|>\left|\bar{h}_{p_{c d}^{-1}\left(t_{3}\right) d}^{-}-\bar{h}_{p_{a b}^{-1}\left(t_{2}\right) b}^{-}\right| .
$$

By the other observation coming from the second part of Lemma 2.3 we also get

$$
\left|\bar{h}_{c p_{c d}^{-1}\left(t_{1}\right)}^{+}-\bar{h}_{a p_{a b}^{-1}\left(t_{1}\right)}^{+}\right|>\left|\bar{h}_{c p_{c d}^{-1}\left(t_{3}\right)}^{+}-\bar{h}_{a p_{a b}^{-1}\left(t_{2}\right)}^{+}\right| .
$$

By Proposition 2.4 the strength of the Poincaré push at $p_{c d}^{-1}\left(t_{2}\right)$ measured within the interval $(c, d)$, i.e. the quantity

$$
\left|\bar{h}_{c p_{c d}^{-1}\left(t_{2}\right)}^{+}+\bar{h}_{p_{c d}^{-1}\left(t_{2}\right) d}^{-}\right|,
$$

is yet smaller than the strength of the Poincaré push at $p_{c d}^{-1}\left(t_{3}\right)$. This completes the proof.

Lemma 2.3. Suppose $0<\beta<1<t$ and $\alpha>1$. Then

$$
\frac{d}{d t}\left(\ln \left(\frac{t^{\alpha}-1}{t-1} \cdot \frac{1}{h^{\prime}(1)}\right)\right)<\frac{d}{d t}\left(\ln \left(\frac{t^{\alpha}-\beta^{\alpha}}{t-\beta} \cdot \frac{1}{h^{\prime}(\beta)}\right)\right)
$$

and also

$$
\frac{d}{d \beta}\left(\ln \left(\frac{1-\beta^{\alpha}}{1-\beta} \cdot \frac{1}{h^{\prime}(1)}\right)\right)>\frac{d}{d \beta}\left(\ln \left(\frac{t^{\alpha}-\beta^{\alpha}}{t-\beta} \cdot \frac{1}{h^{\prime}(t)}\right)\right) .
$$

Proof. The factors $1 / h^{\prime}(1)$ and $1 / h^{\prime}(\beta)$ in $(2.7)$, as well as $1 / h^{\prime}(1)$ and $1 / h^{\prime}(t)$ in (2.8), are put only to conform with the notation of $(2.2)$ and (2.3) and of course can be omitted in computations.

We start with an explicit calculation of the derivatives in (2.7), which leads to

$$
\alpha\left(\frac{t^{\alpha}}{t^{\alpha}-1}-\frac{t^{\alpha}}{t^{\alpha}-\beta^{\alpha}}\right)<\frac{t}{t-1}-\frac{t}{t-\beta} .
$$

For $\alpha=1$, both sides of this inequality coincide, so it will suffice to show that the left hand side is decreasing in $\alpha$. After substitution $x=1 / t, y=\beta / t$, 
the function to be examined is

$$
F(\alpha)=\alpha\left(\frac{1}{1-x^{\alpha}}-\frac{1}{1-y^{\alpha}}\right)
$$

where $0<y<x<1$. Differentiating $F$ in $\alpha$ and again substituting $u=x^{\alpha}$, $v=y^{\alpha}$, we are led to an equivalent inequality

$$
\frac{1}{1-u}+\frac{u \ln u}{(1-u)^{2}}<\frac{1}{1-v}+\frac{v \ln v}{(1-v)^{2}}, \quad 0<v<u<1 .
$$

So we only need to check that the function $\frac{1}{1-u}+\frac{u \ln u}{(1-u)^{2}}$ is decreasing for $u \in(0,1)$, which is elementary.

The proof of (2.8) is similar.

We conclude this section with a lemma, related to Lemma 2.1 and Proposition 2.2, explaining what happens when we change the position of a point within an interval $(a, b)$ of finite nonlinearity, i.e. not bounded by the critical point of $h$. In that case, unlike in the statement of Lemma 2.1, we do not get precise information about the gain in the Poincaré coordinate of the image. Instead, we have a simple inequality.

Lemma 2.4. For any $0<a<x_{2}<x_{1}<b$,

$$
\left|\bar{h}_{a b}\left(p_{a b}\left(x_{2}\right)\right)-\bar{h}_{a b}\left(p_{a b}\left(x_{1}\right)\right)\right|>\left|\bar{h}_{x_{2} b}^{-}-\bar{h}_{x_{1} b}^{-}\right| .
$$

Proof. First, by homogeneity we set $b=h(b)=1$. By (2.1) we have

$$
\left|\bar{h}_{a b}\left(p_{a b}\left(x_{2}\right)\right)-\bar{h}_{a b}\left(p_{a b}\left(x_{1}\right)\right)\right|=\left|p_{h(a) h(b)}\left(h\left(x_{2}\right)\right)-p_{h(a) h(b)}\left(h\left(x_{1}\right)\right)\right| .
$$

Because the critical point of $h$ is farther away from $h(b)$ than the endpoint $h(a)$, the Poincaré length of $\left(h\left(x_{2}\right), h\left(x_{1}\right)\right)$ measured within $(h(a), h(b))$ is larger than that measured within $(0, h(b))$. By Lemma 2.1, the latter is strictly larger that $\left|\bar{h}_{x_{2} b}^{-}-\bar{h}_{x_{1} b}^{-}\right|$, and so we are done.

3. The dynamics. In this section, we make use of the observations from the previous section in a dynamical setting.

For $a=1$, the point 0 is the critical point of period 2 of the map $f_{a}=-|x|^{\alpha}+a$.

We now restrict our attention to positive parameters smaller than 1 . We shall denote by $0_{a}, 1_{a}, 2_{a}, \ldots$ the successive points of the orbit of the critical point of the mapping $f_{a}$. We have the following main

Theorem 3.1. For every positive integer $n$ the ratio function

$$
r_{n}(a):=\frac{\left|(n+2)_{a}-(n+1)_{a}\right|}{\left|(n+1)_{a}-n_{a}\right|}=\frac{\left|f_{a}^{(n+2)}(0)-f_{a}^{(n+1)}(0)\right|}{\left|f_{a}^{(n+1)}(0)-f_{a}^{n}(0)\right|},
$$

where $f_{a}(x)=-h(|x|)+a$, is a strictly increasing function of $a \in(0,1)$. 
Proof. First notice that by homogeneity we may set $0_{a}=0,1_{a}=1$ (i.e. $n_{a} \mapsto n_{a} / 1_{a}$ ), and the dependence on the parameter $a$ translates into the dependence on the value of $2_{a} \in(0,1)$.

Now if $0<u<v<1$, then $2_{u}>2_{v}$, so

$$
\frac{\left|2_{u}-1_{u}\right|}{\left|1_{u}-0_{u}\right|}<\frac{\left|2_{v}-1_{v}\right|}{\left|1_{v}-0_{v}\right|} .
$$

Because the interval $\left(2_{v}, 1\right)$ has larger nonlinearity than $\left(2_{u}, 1\right)$, the strength of the Poincaré push of $2 v$ towards zero under the action of $h$ is larger than that of $2_{u}$. So we obviously have

$$
\frac{\left|3_{v}-2_{v}\right|}{\left|2_{v}-1\right|}>\frac{\left|3_{u}-2_{u}\right|}{\left|2_{u}-1\right|} .
$$

To make the next step we recall Lemma 2.1. We consider a triple of points $\left(2_{v}, 3_{v}, 1\right)$ and the Poincaré push under $\bar{h}_{2_{v} 1}$. The absolute value of this push is by Proposition 2.4 smaller than $\left|\bar{h}_{2_{v} 1}^{-}\right|$(for the map $h$ and the positive orientation of the interval, the value of the limit quantity $\bar{h}^{-}$is negative!). But for our argument this does not suffice. We need to compare the growth of the strength of this push against the increase of the Poincare coordinate of $3_{v}$ within $\left(2_{v}, 1\right)$ to the same quantity for $3_{u}$ in $\left(2_{u}, 1\right)$. That is, we want to see that

$$
\bar{h}_{2_{v} 1}\left(p_{2_{v} 1}\left(3_{v}\right)\right)-\bar{h}_{2_{u} 1}\left(p_{2_{u} 1}\left(3_{u}\right)\right)>0 .
$$

By Lemma 2.1,

$$
p_{2_{v} 1}\left(3_{v}\right)=p_{2_{u} 1}\left(3_{u}\right)+\left|p_{01}\left(2_{v}\right)-p_{01}\left(2_{u}\right)\right|+\left|\bar{h}_{2_{v} 1}^{-}-\bar{h}_{2_{u} 1}^{-}\right|,
$$

so by Proposition 2.2,

$\bar{h}_{2_{v} 1}\left(p_{2_{v} 1}\left(3_{v}\right)\right)=p_{2_{u} 1}\left(3_{u}\right)+\left|p_{01}\left(2_{v}\right)-p_{01}\left(2_{u}\right)\right|+\left|\bar{h}_{2_{v} 1}^{-}-\bar{h}_{2_{u} 1}^{-}\right|+\bar{h}_{3_{v} 1}^{-}+\bar{h}_{2_{v} 3_{v}}^{+}$ whereas

$$
\bar{h}_{2_{u} 1}\left(p_{2_{u} 1}\left(3_{u}\right)\right)=p_{2_{u} 1}\left(3_{u}\right)+\bar{h}_{3_{u} 1}^{-}+\bar{h}_{2_{u} 3_{u}}^{+}
$$

and we get

$$
\begin{aligned}
& \bar{h}_{2_{v} 1}\left(p_{2_{v} 1}\left(3_{v}\right)\right)-\bar{h}_{2_{u} 1}\left(p_{2_{u} 1}\left(3_{u}\right)\right) \\
& \quad=\left|p_{01}\left(2_{v}\right)-p_{01}\left(2_{u}\right)\right|+\left|\bar{h}_{3_{v} 1}^{-}+\bar{h}_{2_{v} 3_{v}}^{+}-\bar{h}_{2_{v} 1}^{-}\right|-\left|\bar{h}_{3_{u} 1}^{-}+\bar{h}_{2_{u} 3_{u}}^{+}-\bar{h}_{2_{u} 1}^{-}\right| .
\end{aligned}
$$

By Proposition 2.4,

$$
\left|\bar{h}_{3_{v} 1}^{-}+\bar{h}_{2_{v} 3_{v}}^{+}-\bar{h}_{2_{v} 1}^{-}\right|>\left|\bar{h}_{p_{2_{v} 1}^{-1}\left(p_{2_{u} 1}\left(3_{u}\right)\right) 1}^{-}+\bar{h}_{2_{v} p_{2_{v} 1}^{-1}\left(p_{2_{u} 1}\left(3_{u}\right)\right)}^{+}-\bar{h}_{2_{v} 1}^{-}\right|
$$

and by Proposition 2.5 the latter quantity is greater than $\left|\bar{h}_{3_{u} 1}^{-}+\bar{h}_{2_{u} 3_{u}}^{+}-\bar{h}_{2_{u} 1}^{-}\right|$, so (3.2) is proven. The conclusion of the above argument is

$$
p_{3_{v} 2_{v}}\left(4_{v}\right)-p_{3_{u} 2_{u}}\left(4_{u}\right)>\left|p_{01}\left(2_{v}\right)-p_{01}\left(2_{u}\right)\right|>0,
$$


which in particular yields

$$
\frac{\left|4_{v}-3_{v}\right|}{\left|3_{v}-2_{v}\right|}>\frac{\left|4_{u}-3_{u}\right|}{\left|3_{u}-2_{u}\right|}
$$

Now Lemma 2.4 will enable us to proceed recursively.

Let $n$ be a positive even integer and suppose we already know that the nonlinearity of the interval $\left((n-2)_{v},(n-1)_{v}\right)$ is larger than that of $\left((n-2)_{u},(n-1)_{u}\right)$, and also that $p_{(n-1)_{v}(n-2)_{v}}\left(n_{v}\right)>p_{(n-1)_{u}(n-2)_{u}}\left(n_{u}\right)$. Then by Propositions 2.4 and 2.5 it is clear that, similarly to the argument for (3.1), the inequality

$$
\frac{\left|j_{v}-(j-1)_{v}\right|}{\left|(j-1)_{v}-(j-2)_{v}\right|}>\frac{\left|j_{u}-(j-1)_{u}\right|}{\left|(j-1)_{u}-(j-2)_{u}\right|}
$$

is satisfied not only for $j=n$, but also for the odd number $j=n+1$. Thus the interval $\left(n_{v},(n+1)_{v}\right)$ has larger nonlinearity than $\left(n_{u},(n+1)_{u}\right)$. To complete the inductive step from $n$ to $n+2$ we only need to check that $p_{(n+1)_{v} n_{v}}\left((n+2)_{v}\right)>p_{(n+1)_{u} n_{u}}\left((n+2)_{u}\right)$. Proceeding as in the proof of $(3.2)$ (but this time without Lemma 2.1 in place) we get

$$
\begin{aligned}
p_{n_{v}(n-1)_{v}} & \left((n+1)_{v}\right) \\
= & p_{n_{u}(n-1)_{u}}\left((n+1)_{u}\right)+\left(p_{(n-1)_{v}(n-2)_{v}}\left(n_{v}\right)-p_{(n-1)_{u}(n-2)_{u}}\left(n_{u}\right)\right) \\
+ & {\left[\left(\bar{h}_{(n-1)_{v}(n-2)_{v}}\left(p_{(n-1)_{v}(n-2)_{v}}\left(n_{v}\right)\right)-p_{(n-1)_{v}(n-2)_{v}}\left(n_{v}\right)\right)\right.} \\
& \left.-\left(\bar{h}_{(n-1)_{u}(n-2)_{u}}\left(p_{(n-1)_{u}(n-2)_{u}}\left(n_{u}\right)\right)-p_{(n-1)_{u}(n-2)_{u}}\left(n_{u}\right)\right)\right] .
\end{aligned}
$$

Thus, by Proposition 2.2,

$$
\begin{aligned}
\bar{h}_{n_{v}(n-1)_{v}} & \left(p_{n_{v}(n-1)_{v}}\left((n+1)_{v}\right)\right) \\
= & p_{n_{u}(n-1)_{u}}\left((n+1)_{u}\right)+\left(p_{(n-1)_{v}(n-2)_{v}}\left(n_{v}\right)-p_{(n-1)_{u}(n-2)_{u}}\left(n_{u}\right)\right) \\
+ & {\left[\left(\bar{h}_{(n-1)_{v}(n-2)_{v}}\left(p_{(n-1)_{v}(n-2)_{v}}\left(n_{v}\right)\right)-p_{(n-1)_{v}(n-2)_{v}}\left(n_{v}\right)\right)\right.} \\
& -\left(\bar{h}_{(n-1)_{u}(n-2)_{u}}\left(p_{(n-1)_{u}(n-2)_{u}}\left(n_{u}\right)\right)-p_{(n-1)_{u}(n-2)_{u}}\left(n_{u}\right)\right) \\
& +\bar{h}_{(n+1)_{v}(n-1)_{v}}^{-}+\bar{h}_{n_{v}(n+1)_{v}}^{+}
\end{aligned}
$$

and finally

$$
\begin{aligned}
& \bar{h}_{n_{v}(n-1)_{v}}\left(p_{n_{v}(n-1)_{v}}\left((n+1)_{v}\right)\right)-\bar{h}_{n_{u}(n-1)_{u}}\left(p_{n_{u}(n-1)_{u}}\left((n+1)_{u}\right)\right) \\
&=[\left(p_{(n-1)_{v}(n-2)_{v}}\left(n_{v}\right)-p_{(n-1)_{u}(n-2)_{u}}\left(n_{u}\right)\right) \\
&+\left(\bar{h}_{(n-1)_{v}(n-2)_{v}}\left(p_{(n-1)_{v}(n-2)_{v}}\left(n_{v}\right)\right)-p_{(n-1)_{v}(n-2)_{v}}\left(n_{v}\right)\right) \\
&\left.-\left(\bar{h}_{(n-1)_{u}(n-2)_{u}}\left(p_{(n-1)_{u}(n-2)_{u}}\left(n_{u}\right)\right)-p_{(n-1)_{u}(n-2)_{u}}\left(n_{u}\right)\right)\right] \\
&+\left[\bar{h}_{(n+1)_{v}(n-1)_{v}}^{-}+\bar{h}_{n_{v}(n+1)_{v}}^{+}-\bar{h}_{(n+1)_{u}(n-1)_{u}}^{-}-\bar{h}_{n_{u}(n+1)_{u}}^{+}\right] .
\end{aligned}
$$


By Proposition 2.4 the second summand in brackets is larger than

$$
\begin{array}{r}
\bar{h}_{p_{n_{v}(n-1)_{v}}^{-1}\left(p_{n_{u}(n-1)_{u}}\left((n+1)_{u}\right)\right)(n-1)_{v}}+\bar{h}_{n_{v} p_{n_{v}(n-1)_{v}}^{-1}\left(p_{n_{u}(n-1) u}\left((n+1)_{u}\right)\right)}^{-} \\
-\bar{h}_{(n+1)_{u}(n-1) u}^{-}-\bar{h}_{n_{u}(n+1)_{u}}^{+},
\end{array}
$$

and this, by Proposition 2.5, is in turn larger than $\bar{h}_{n_{v}(n-1)_{v}}^{-}-\bar{h}_{n_{u}(n-1)_{u}}^{-}$, a negative quantity. However, by Lemma 2.4 the absolute value of this quantity is smaller than the absolute value of the first summand in brackets in (3.4). This is so because

$$
\begin{aligned}
{\left[\left(p_{(n-1)_{v}(n-2)_{v}}\left(n_{v}\right)-p_{(n-1)_{u}}(n-2)_{u}\right.\right.} & \left.\left(n_{u}\right)\right) \\
& +\left(\bar{h}_{(n-1)_{v}(n-2)_{v}}\left(p_{(n-1)_{v}(n-2)_{v}}\left(n_{v}\right)\right)-p_{(n-1)_{v}(n-2)_{v}}\left(n_{v}\right)\right) \\
& \left.-\left(\bar{h}_{(n-1)_{u}(n-2)_{u}}\left(p_{(n-1)_{u}(n-2)_{u}}\left(n_{u}\right)\right)-p_{(n-1)_{u}(n-2)_{u}}\left(n_{u}\right)\right)\right] \\
= & \left(p_{(n-1)_{v}(n-2)_{v}}\left(n_{v}\right)-p_{(n-1)_{u}(n-2)_{u}}\left(n_{u}\right)\right) \\
& +\left(\bar{h}_{(n-1)_{v}(n-2)_{v}}\left(p_{(n-1)_{u}(n-1)_{u}}\left(n_{u}\right)\right)\right. \\
& \left.-\bar{h}_{(n-1)_{u}(n-2)_{u}}\left(p_{(n-1)_{u}(n-2)_{u}}\left(n_{u}\right)\right)\right) \\
& +\left[\left(\bar{h}_{(n-1)_{v}(n-2)_{v}}\left(p_{(n-1)_{v}(n-2)_{v}}\left(n_{v}\right)\right)-p_{(n-1)_{v}(n-2)_{v}}\left(n_{v}\right)\right)\right. \\
& \left.-\left(\bar{h}_{(n-1)_{v}(n-2)_{v}}\left(p_{(n-1)_{u}(n-2)_{u}}\left(n_{u}\right)\right)-p_{(n-1)_{u}(n-2)_{u}}\left(n_{u}\right)\right)\right]
\end{aligned}
$$

and by Lemma 2.4,

$$
\begin{aligned}
\left(p_{(n-1)_{v}(n-2)_{v}}\right. & \left.\left(n_{v}\right)-p_{(n-1)_{u}(n-2)_{u}}\left(n_{u}\right)\right) \\
& +\left[\left(\bar{h}_{(n-1)_{v}(n-2)_{v}}\left(p_{(n-1)_{v}(n-2)_{v}}\left(n_{v}\right)\right)-p_{(n-1)_{v}(n-2)_{v}}\left(n_{v}\right)\right)\right. \\
& \left.-\left(\bar{h}_{(n-1)_{v}(n-2)_{v}}\left(p_{(n-1)_{u}(n-2)_{u}}\left(n_{u}\right)\right)-p_{(n-1)_{u}(n-2)_{u}}\left(n_{u}\right)\right)\right] \\
> & \mid \bar{h}_{n_{v}(n-1)_{v}}^{-}-\bar{h}_{p_{(n-1)_{v}(n-2)_{v}}^{-1}}\left(p_{(n-1)_{u}(n-2) u}\left(n_{u}\right)\right)(n-1)_{v}
\end{aligned}
$$

whereas

$$
\begin{aligned}
& \bar{h}_{(n-1)_{v}(n-2)_{v}}\left(p_{(n-1)_{u}(n-2)_{u}}\left(n_{u}\right)\right)-\bar{h}_{(n-1)_{u}(n-2)_{u}}\left(p_{(n-1)_{u}(n-2)_{u}}\left(n_{u}\right)\right) \\
& \left.=\left(\bar{h}_{p_{(n-1)_{v}(n-2)_{v}}^{-1}\left(p_{(n-1) u}(n-2)_{u}\right.}\left(n_{u}\right)\right)(n-1)_{v}-\bar{h}_{n_{u}(n-1)_{u}}^{-}\right) \\
& +\left(\bar{h}_{(n-2)_{v} p_{(n-1)_{v}(n-2)_{v}}^{-1}\left(p_{(n-1)_{u}(n-2)_{u}}\left(n_{u}\right)\right)}-\bar{h}_{(n-2)_{u} n_{u}}^{+}\right) \\
& >\bar{h}_{p_{(n-1)_{v}(n-2) v}^{-1}}^{-}\left(p_{(n-1) u(n-2)_{u}}\left(n_{u}\right)\right)(n-1)_{v}-\bar{h}_{n_{u}(n-1)_{u}}^{-} .
\end{aligned}
$$

This completes the inductive step.

So we have proved that when we increase the parameter the outcome of any two consecutive steps constitutes a larger fraction of a "more nonlinear" interval. Thus, this newly obtained domain itself has larger nonlinearity and the above procedure can be applied repeatedly, taking consecutive intervals 
delimited by the post-critical orbit as new domains of $h$ each time. The proof of Theorem 3.1 is now complete.

\section{References}

[1] S. Dawson, R. Galeeva, J. Milnor and C. Tresser, A monotonicity conjecture for real cubic maps, in: Real and Complex Dynamical Systems, NATO Adv. Sci. Inst. Ser. C Mat. Phys. Sci. 464, Kluwer, Dordrecht, 1995, 165-187.

[2] V. Dragan, A. Jones and P. Stacey, Repeated radicals and the real Fatou theorem, Austral. Math. Soc. Gaz. 29 (2002), 259-268.

[3] J. Graczyk and G. Świątek, Induced expansion for quadratic polynomials, Ann. Sci. École Norm. Sup. 29 (1996), 399-482.

[4] J. Milnor and W. Thurston, Iterated maps of the interval, in: Dynamical Systems, Lecture Notes in Math. 1342, Springer, Berlin, 1988, 465-563.

[5] W. Pałuba, On conjugacies of infinitely renormalizable maps, Ph.D. thesis, City Univ. of New York, New York, 1992.

[6] D. Sullivan, Bounds, quadratic differentials, and renormalization conjectures, in: AMS Centennial Publications, Vol. 2, AMS, Providence, RI, 1992, 417-466.

[7] M. Tsujii, A simple proof for monotonicity of entropy in the quadratic family, Ergodic Theory Dynam. Systems 20 (2000), 925-933.

Waldemar Pałuba

Institute of Mathematics

University of Warsaw

Banacha 2

02-097 Warszawa, Poland

E-mail: paluba@mimuw.edu.pl

Received February 18, 2004;

received in final form October 11, 2004 